\title{
La implementación del taller "Juguemos con Álgebra" para la mejora del rendimiento académico en el tema de expresiones algebraicas y la resolución de ecuaciones lineales, así como la conceptualización de la materia de matemáticas de los estudiantes de $3^{\circ}$ de secundaria
}

\author{
Ana Laura Núñez Rodríguez, Alma Minerva Aguilar Martínez, Mireya Sarahí Abarca Cedeño, \\ Jesús Antonio Larios Trejo \\ Universidad de Colima, México
}

\begin{abstract}
Resumen: Con frecuencia, el diseño de actividades dinámicas y atractivas para la enseñanza de las matemáticas es uno de los principales retos a los que se enfrentan los profesores de dicha materia, debido a que el Programa de Estudios 2011 del Sistema Educativo Mexicano considera que es el docente quien debe propiciar situaciones didácticas para despertar el interés en los alumnos; promoviendo el desarrollo de ciertas habilidades, tanto a través del trabajo individual y autónomo como colectivo. Buscando diseñar y validar experiencias que favorezcan el proceso de enseñanza-aprendizaje de las matemáticas, se llevó a cabo la presente investigación, la cual tuvo como objetivo evaluar el impacto del taller "Juguemos con Álgebra" en la conceptualización de la materia de matemáticas en alumnos de $3^{\circ}$ grupo A de la escuela secundaria Alberto Larios Villalpando, turno vespertino, así como también el impacto en el rendimiento académico en el tema de expresiones algebraicas y resolución de ecuaciones lineales. En dicho taller se puso en práctica lo propuesto en el Programa de Estudios 2011, es decir, trabajar con base en un enfoque didáctico, el cual consiste en problematizar a los alumnos para que discutan y analicen sus procedimientos y resultados, apoyando esto con el juego, como recurso para la enseñanza del álgebra.
\end{abstract}

Palabras clave: enseñanza de matemáticas, álgebra, redes semánticas, rendimiento académico, taller recreativo, conceptualización, alumnos de secundaria

\begin{abstract}
Often, the design of dynamic and engaging activities for teaching mathematics is one of the main challenges faced by teachers of the subject, because the Studies Program 2011 of Mexican Educational System considers that is the teacher who should promote teaching situations to arouse interest in students, promoting the development of skills, by working both individually and as a collective. Looking to design and validate experiences that promote the teaching and learning of mathematics, was conducted this research, which aimed to assess the impact of the workshop "Juguemos con Álgebra" in the conceptualization of the subject of mathematics students of 3rd grade group A, of high school Alberto Larios Villalpando, as well as the impact on academic performance in the area of algebraic expressions and solving linear equations. The workshop implemented the Program of Studies 2011, that is, work-based training approach, which is to problematize students to discuss and analyze their procedures and results, supporting this with the game, as a resource for teaching of algebra.
\end{abstract}

Keywords: Teaching Math, Algebra, Semantic Networks, Academic Performance, Recreational Workshop, Conceptualization, High School Students

Revista Internacional de Educación y Aprendizaje

Volumen 2, Número $2<\mathrm{http}$ ://sobrelaeducacion.com/>, ISSN 2255-453X

(C) Global Knowledge Academics. Ana Laura Núñez Rodríguez et al.

Todos los derechos reservados. Permisos: soporte@gkacademics.com 


\section{Planteamiento del problema}

$\mathrm{M}$ atemáticas es una materia a la que la gran mayoría de los alumnos, especialmente en secundaria, le tienen cierto rechazo debido a las experiencias que han venido teniendo en el transcurso de su vida escolar, lo cual se infiere a través de expresiones cotidianas de los estudiantes en las que expresan las dificultades que experimentan al abordar la asignatura, e incluso los bajos rendimientos obtenidos en pruebas nacionales estandarizadas. El problema se agranda cuando llegan a segundo grado de secundaria, al comenzar a tener mayor cercanía con el tema de álgebra; al mismo tiempo su grado de dificultad comienza a aumentar provocando a menudo confusión y creando en ellos grandes interrogantes sobre el porqué de su expresión y el para qué de su implementación, y es ahí cuando en la mayoría de los casos se termina de perder el poco interés que se tenía por la materia y con esto perjudicar a su rendimiento académico, e incluso el desempeño en otras asignaturas que requieran del óptimo manejo de los números, y yendo más allá, su aplicación eficiente para resolver problemas cotidianos. Lo anterior se pone de manifiesto en los resultados de la prueba ENLACE 2012 (prueba estandarizada que evalúa el rendimiento académico de los estudiantes en México), en la cual la gran mayoría de las escuelas generales del estado de Colima se encuentran con puntajes que apenas sobrepasan la media; este examen maneja calificaciones que van de 200 puntos (si alguien no tuviera ningún acierto) y un máximo de 800 puntos (poner una nota de algún resultado de referencia).

Por lo ya mencionado, buscando estrategias eficientes que contribuyan a la solución de este problema, se desarrolló el presente proyecto, buscando responder a la pregunta: ¿cómo influye el taller "Juguemos con Álgebra" en el rendimiento académico de los estudiantes de $3^{\circ}$ de secundaria en el tema de expresiones algebraicas y resolución de ecuaciones lineales, así como en la conceptualización que se tiene de la materia de matemáticas?

\section{Referentes teóricos}

Para lograr el objetivo del trabajo de investigación es necesario definir los temas clave que sustentan la importancia del desarrollo del mismo, desde las matemáticas como disciplina y el proceso educativo implícito.

\section{Matemáticas en la educación}

A pesar de la relevancia y la cotidianidad de la asignatura de matemáticas, actualmente en la educación secundaria, uno de los principales retos que enfrentan los decentes es que los alumnos no logran observar por qué estudiar dicha disciplina, o en otras palabras, no encuentran su utilidad en la vida real; quizá esto pueda deberse a la forma en que los maestros presentan los contenidos programáticos, alejándolos de la realidad. Ahí la importancia de la acción docente, donde su labor principal deberá enfocarse en la transformación de la matemática pura a una matemática comprensible y manipulable por los alumnos, con un fuerte referente en su aplicación, para que los mismos estudiantes sean los que generen y se apropien de los conocimientos, logrando así la obtención de aprendizajes significativos. Con este enfoque, los Programas de Estudio 2011, en la guía para el maestro de matemáticas (SEP, 2011) ha venido haciendo diversas modificaciones, con el propósito de lograr esta transformación hacia una asignatura relevante y útil.

A continuación se presenta lo planteado en el programa de matemáticas de educación básica establecidas por la Secretaría de Educación Pública (SEP). 


\section{Programa de Matemáticas de Educación Básica en México}

El nuevo programa de estudio de matemáticas 2011 en México, tiene como objetivo "el desarrollo de competencias con el fin de que cada estudiante pueda desenvolverse en una sociedad que le demanda nuevos desempeños para relacionarse en un marco de pluralidad y democracia, y en un mundo global e interdependiente" (SEP, 2011: 8), esto significa, que a través de este nuevo diseño, se pretende que los alumnos sean capaces de desenvolverse no solo en el ámbito escolar, sino también como persona y miembros de una sociedad.

"La experiencia que vivan los alumnos al estudiar matemáticas en la escuela puede traer como consecuencias: el gusto o el rechazo por ellas, la creatividad para buscar soluciones o la pasividad para escucharlas y tratar de reproducirlas" (SEP, 2011: 19) por ende, se pretende que los maestros sean los que generen situaciones didácticas donde los alumnos muestren su ingenio y creatividad para establecer estrategias que los encaminen hacia un aprendizaje significativo, aplicables a su vida real.

Hablando ya de los contenidos del Programa de Estudios 2011, sabemos que se encuentra organizado en cuatro ejes temáticos, que son; 1) sentido numérico y pensamiento algebraico, 2) forma, espacio y medida, 3) manejo de la información y 4) actitud hacia el estudio de las matemáticas, este último es nuevo dentro del programa, ya que en el 2006 no estaba incluido como tal.

Debido a que la investigación se centrará en el tema propiamente de álgebra, los ejes temáticos a fortalecer serán los referentes a: 1) sentido numérico y pensamiento algebraico y 4) actitud hacia el estudio de las matemáticas; donde el desarrollo del trabajo tanto individual como en equipo serán un aspecto clave para el logro del aprendizaje.

Ahora bien, si regresamos a las experiencias vividas dentro del aula, nos hemos dado cuenta que la base del álgebra es principalmente el buen uso y manejo de las expresiones algebraicas para la resolución de diversas situaciones a través de ecuaciones, por lo anterior, la importancia de implementar el taller "Juguemos con Álgebra", donde se manejan los temas: expresiones algebraicas y resolución de ecuaciones lineales. Por estas razones es conveniente ver a qué hace referencia el concepto de álgebra.

\section{Álgebra en secundaria}

“(Del árabe al-gabr, la restauración). Forma parte de las matemáticas, trata de cantidades generales, sirviéndose para representarlas, de letras u otros signos especiales" (Jiménez, Rodríguez y Estrada, 2006:131). Si observamos a detalle esta definición, podemos verificar que el lenguaje algebraico es la base de dicha disciplina, puesto que la finalidad de manejar literales y símbolos son propiamente del lenguaje algebraico, por otra parte asociándolo a esto, podemos decir que existe una restauración al convertir del lenguaje común al algebraico ya que es la misma expresión solo que representada de otra manera (es decir, del texto a una expresión algebraica).

En el libro del maestro de matemáticas de educación secundaria, nos dice que el tema de álgebra "más que cualquier otra parte de las matemáticas en la educación secundaria, representa la transición entre la aritmética y la geometría elementales de la primaria" (Alarcón, Bonilla, Nava, Rojano y Quintero, 1994: 123) por tal motivo, es que a nivel secundaria inician las confusiones del por qué combinar letras con números, y no se comprende el significado que estas toman, y peor aun cuando no se traen las bases necesarias de la geometría y la aritmética que debieron adquirirse en primaria.

Los alumnos al tener sus primeros inicios con el álgebra, comienzan a preguntarse acerca de la importancia de su estudio, sin comprender que "casi todas las matemáticas de la preparatoria y la universidad requieren del lenguaje del álgebra para modelar situaciones y resolver problemas, así como para expresar conceptos y operar con ellos en niveles cada vez más abstractos" (Alarcón et al., 1994: 123). 
Ahora bien, ya que el lenguaje algebraico es la base del álgebra, en el siguiente apartado se revisarán algunas de las dificultades de éste a nivel secundaria.

\section{Lenguaje algebraico en secundaria}

Antes de comenzar a hablar acerca del lenguaje algebraico a nivel secundaria, es importante definir lo que es el lenguaje algebraico.

En álgebra, están contenidos números, letras que representan números y expresiones que equivalen a determinadas cantidades; todo esto hace referencia al lenguaje algebraico, el cual se utiliza en dos sentidos: para representar algebraicamente los enunciados ordinarios y para expresar de manera verbal o escrita las expresiones algebraicas (Jiménez et al., 2006), situación que con base a la experiencia produce ciertas dificultades en los alumnos de secundaria, debido a que no encuentran esa relación con su vida diaria y sin poder entender que "a diario el hombre usa formas y modelos como parte del lenguaje que utiliza para comunicarse" (Jiménez et al., 2006: 27), por ejemplo un conductor, que debe conocer la simbología de las leyes de tránsito.

Por otro lado existen algunas habilidades a desarrollar en los alumnos de secundaria relacionadas con el lenguaje algebraico. Socas (2008, como se citó por Sánchez, Gómez, y de la Cruz, 2010) considera que las habilidades algebraicas pertinentes que debe poseer un alumno de educación básica son:

- Habilidad para aplicar los conocimientos algebraicos a la resolución de problemas

- Habilidad para usar el lenguaje algebraico en la comunicación de ideas

- Habilidad para razonar el lenguaje algebraico

- Habilidad para el conocimiento y el entendimiento de procedimientos algebraicos

- Actitud de los alumnos en el trabajo individual y cooperativo y en su apreciación al álgebra.

Como podemos apreciar, el lenguaje algebraico debe ser el primer paso a dominar por los alumnos de secundaria para poder desarrollar dichas habilidades, las cuales pretenden facilitar en estos mismos su nivel de comprensión ante algo que quizá suela ser un poco abstracto en esta etapa.

Si se logra comprender el lenguaje algebraico, y así mismos el desarrollo de dichas habilidades, se propiciará de alguna manera a la mejora del rendimiento académico, el cual se aborda a continuación.

\section{Rendimiento académico}

El rendimiento académico se entiende como "el producto que da el alumnado en los centros de enseñanza y que habitualmente se expresa a través de las calificaciones escolares" (Martínez, 2007: 34), sin embargo, hablar de rendimiento académico no implica solamente un número, ya que al hablar de este se pretende hacer alusión a los logros académicos alcanzados durante un periodo de tiempo determinado.

El actual programa de estudios, vigente desde el 2011, propone un nuevo enfoque didáctico que sugiere que los alumnos adquieran conocimientos y habilidades con sentido y significado a través de la problematización, para lo cual maneja el trabajo colaborativo como una estrategia de enseñanza aprendizaje, haciendo a un lado el enfoque tradicionalista y propiciando en los alumnos la interacción constante para el intercambio de ideas y conocimientos.

Generalmente para conocer el rendimiento académico de los alumnos, los maestros asignan diversos criterios de evaluación en los cuales entran el cumplimiento de las tareas, el trabajo en clase, las participaciones, los exámenes elaborados por los mismos profesores y en algunos casos las asistencias, por todo esto podemos notar que la calificación asignada al final de un curso recae solamente en la responsabilidad del mismo estudiante, dejando a un lado la parte de la colaboración entre maestros, padres de familia, alumnos e institución. Además sería conveniente 
decir que el hecho de asignar diversos criterios para evaluar el rendimiento académico de los alumnos, podría considerarse un poco injusto ya que pondría en desventaja a algunos de ellos, esto debido a los distintos contextos en los que se ven inmersos cada uno de los estudiantes.

Actualmente existe una prueba estandarizada llamada ENLACE que pretende medir el rendimiento académico de los estudiantes de todos los centros educativos, la cual hace partícipe a alumnos, profesores, institución y padres de familia.

\section{Prueba ENLACE}

La Evaluación Nacional de Logro Académico en Centros Escolares (ENLACE) es una prueba del Sistema Educativo Nacional que se aplica a planteles públicos y privados del país (SEP, 2012); tiene como finalidad el comparar el rendimiento académico de los alumnos de todas las escuelas del país desde tercer grado de primaria hasta tercero de secundaria y actualmente el último año de bachillerato. Dicha prueba evalúa temas de español, matemáticas y una tercera que varía cada año y se repite cada cuatro años. Debido a los objetivos de esta investigación sólo nos quedaremos con lo referente a la materia de matemáticas.

La prueba ENLACE es un instrumento estandarizado, objetivo, de alcance nacional, diseñado para que los docentes, autoridades educativas, investigadores y escolares de todo el país, dispongan de una medida válida, objetiva y confiable, del estado actual del rendimiento académico de los estudiantes de educación básica (SEP, 2012), por lo cual es sabido que dichos resultados, así como el instrumento, son utilizados para diversas investigaciones educativas con el fin de analizar qué es lo que está pasando en los centros educativos del país. Así mismo, ENLACE podría ser considerada como una estrategia para la mejora de la educación en México debido a que sus contenidos a evaluar van de la mano con los planes y programas de estudio vigentes.

Es importante mencionar que los resultados obtenidos por los alumnos en la prueba ENLACE no influyen en sus calificaciones, además de que la puntuación obtenida en la prueba ENLACE se establece de la siguiente manera: ENLACE otorga 200 puntos por el simple hecho de realizar la prueba, aunque no se obtenga ningún acierto, y un máximo de 800 puntos tomando como media 500 puntos.

\section{Actividad lúdica}

Las personas no aprendemos solas, si no que estamos integradas en un contexto social que da sentido a lo que aprendemos. Este contexto es el que puede hacerle sentir necesidad de lo que falta por aprender y de lo que hay que ajustar en el proceso de aprendizaje. (Barnett, 2003: 92)

Por lo anterior, podemos decir que para reforzar el aprendizaje, el juego es considerado como un recurso para la enseñanza, donde los alumnos interactúan y se divierten, al mismo tiempo que aprenden. Por lo cual en el siguiente apartado abordaremos lo relacionado con el juego como recurso para enseñar matemáticas.

\section{El juego: un recurso para enseñar matemáticas}

Se pretende tomar al juego como recurso para la enseñanza de matemáticas, por lo cual se considera necesario conocer la definición de "juego":

La palabra juego proviene del vocablo latino iocus, que para los romanos significó broma, alegría, jolgorio o diversión. El diccionario de las Ciencias de la Educación lo define como: actividad lúdica que comporta un fin en sí misma, con la independencia de que en ocasiones se realice con un valor extrínseco (García, 2002: 15). 
Respecto a la definición anterior podemos mencionar que esta estrategia debe tener un fin útil para el alumno de manera agradable, para que este sea capaz de otorgarle un valor significativo en su aprendizaje de acuerdo a la interacción que tenga con sus compañeros y no ser solo una situación para pasar el rato y mucho menos para perder el tiempo.

Según García (1998, como se citó por García, 2002), visto desde el punto psicopedagógico, el juego se valora como un elemento que favorece a todo tipo de aprendizajes, puede proporcionar al niño una gran variedad de experiencias y estímulos, todo un bagaje de vivencias útiles y necesarias para su adecuado desarrollo (p. 17).

Dicho esto, podemos relacionarlo con el trabajo colaborativo debido a que el alumno al momento de interactuar con sus compañeros adquiere experiencias que complementan su aprendizaje, convirtiendo al juego en una herramienta para su desarrollo.

Por todo lo anterior, el juego puede ser considerado por los maestros como un recurso para despertar el interés en los alumnos al aprender de una manera distinta a la que están acostumbrados, por lo cual el manejar actividades lúdicas o convertir por lo menos una clase de la materia en un taller recreativo propiciaría el interés y el fortalecimiento de contenidos abordados a través del juego.

Para justificar la importancia que un taller recreativo pudiera tener en el rendimiento académico de los alumnos abordaremos el siguiente apartado.

\section{Taller recreativo}

Para comprender lo referente a este apartado será necesario definir por separado lo relacionado a taller y recreación.

El término taller hace referencia a la creación de "numerosas experiencias que aluden a una trasformación total o parcial del ámbito escolar y son de gran interés por lo que suponen de reflexión activa, mejora y dinamismo en la labor pedagógica cotidiana" (Trueba, 1999: 15).

Wiswell (como se citó por Chacón, 2005) define la recreación como “...una vivencia de disfrute y desarrollo para la promoción del ser humano y su potencialización, es decir proyección al máximo de sus habilidades, capacidades, aptitudes y destrezas, reconociéndolo como un acto fundamental de su proyecto de vida particular y comunitario." (p. 131)

Una vez definidos estos términos podemos decir que un taller recreativo será aquel donde se obtengan experiencias significativas por los alumnos, debido a que el ambiente escolar sufre una transformación al momento de brindar un espacio donde estos desarrollan habilidades, capacidades, actitudes y destrezas, dejando de lado la búsqueda de un numero o calificación a cambio de experiencias satisfactorias generadas a partir de la interacción entre un grupo determinado.

Por todo lo que conlleva un taller recreativo podemos decir que la importancia que este tiene en el rendimiento académico de los alumnos es debido a la implementación del trabajo colaborativo, la interacción y el constante intercambio de ideas, experiencias y conocimientos, reforzándolos así de manera individual y grupal a través del juego como recurso para la enseñanza, además de que gracias a este los alumnos tienden a ser autónomos y construyen sus propias estrategias y analizan cuidadosamente sus resultados.

Con la implementación de este tipo de talleres se propicia a la modificación de ideas, debido a que en el momento en que se implementa una actividad lúdica el ambiente de trabajo deja de ser monótono para convertirse en algo innovador y divertido, sin dejar de lado su propósito fundamental que es propiciar en los alumnos un aprendizaje significativo. Dicho lo anterior se abordará la conceptualización, que hace referencia a la perspectiva que tienen los alumnos, en este caso, de la materia de matemáticas. 


\section{Conceptualización matemática}

La palabra conceptualizar hace referencia a lo siguiente:

Quiere decir que no alcanza con definir algo, decir lo que ese algo es, sino poder darle un contexto, un significado y una valoración. Poder darle un contexto a una idea, la vincula con la realidad y por ende con el hacer. Darle un significado implica la actividad intelectual, comprender (Stigol, 2012, párr. 4).

Apoyándonos con esta definición, podemos decir que en nuestra experiencia educativa ha sido muy común encontrarnos con alumnos que tienen una conceptualización negativa hacia las matemáticas debido a sus experiencias dentro de la escuela, o cuando los maestros no logran despertar en ellos el interés por la materia. A lo anterior también recae la importancia de los talleres recreativos como parte del curso tratando de que los alumnos logren visualizar su importancia y utilidad en la vida diaria, modificando así dicha conceptualización.

Una manera de encontrar el significado ante las diferentes maneras de percibir un concepto, en este caso de la materia de matemáticas, son las redes semánticas, por lo cual se desarrollan a continuación.

\section{Redes semánticas naturales}

Para dejar claro lo que son y el uso adecuado de las redes semánticas naturales es importante citar lo siguiente:

La técnica de las redes semánticas naturales inicialmente propuesta por Figueroa, Gonzales y Solís (1981b) que tiene como propósito fundamental el aproximarse al estudio del significado de manera 'natural', es decir, directamente con los individuos. (Valdez, 2004: 81).

Como sabemos, esta técnica pretende obtener una serie de palabras que definan un concepto, con la finalidad de encontrar el significado que esta tiene para un individuo. Para lo cual, se organiza una ficha impresa con la palabra a definir y dos columnas de cinco renglones para que el o los individuos coloquen en una de ellas las palabras definidoras y en la otra columna las jerarquías contribuidas a las mismas.

Cuando estas se trabajan en un grupo determinado es conveniente reunir las palabras y tomar las 15 con mayor valor $\mathrm{M}$ total (frecuencia por valor semántico). Para después pasar a observar la conceptualización que tuvo dicho grupo con relación a la palabra dada.

Para hacer el agrupamiento y selección de las 15 palabras, se realiza una tabla donde se anotan estas con el número de frecuencias de aparición, el cual se multiplica por un valor semántico asignado, donde a las palabras con valor jerárquico de 1 se le da un valor semántico de 10 , a las de 2 una valor de 9 , a las de 3 un valor de 8 , a las de 4 un valor de 7 y por ultimo a las de valor jerárquico de 5 se les asigna un valor semántico de 6 , dichos valores se multiplican, sumando así el valor M total.

Los cuatro principales valores o resultados, con los que se puede analizar la información que fue generada por los sujetos son:

- Valor J, resulta del total de palabras definidoras que fueron generadas por los sujetos para definir el estímulo en cuestión.

- Valor M, se obtiene de la multiplicación que se hace de la frecuencia de aparición por la jerarquía obtenida para cada una de las palabras definidoras generadas por los sujetos.

- Valor FMG, se obtiene para todas las palabras definidoras que conformaron el conjunto SAM, a través de una sencilla regla de tres, tomando como punto de par- 
tida que la palabra definidora con el valor $\mathrm{M}$ más grande, representará el 100\%. Este valor es un indicador en términos de porcentajes.

- Conjunto SAM, grupo de las 15 palabras definidoras que obtuvieron los mayores valores M totales (Valdez, 2004: 86).

\section{Método}

\section{Objetivos}

\section{General:}

Evaluar el impacto del taller "Juguemos con Álgebra" en el rendimiento académico en el tema de expresiones algebraicas y resolución de ecuaciones lineales, así como en la conceptualización de la materia de matemáticas en los alumnos de $3^{\circ}$ de secundaria.

Especificos:

1. Identificar el rendimiento académico de alumnos de $3^{\circ}$ a través de un pre-test creado con reactivos de la prueba ENLACE 2012.

2. Conocer la conceptualización de la materia de matemáticas en alumnos de $3^{\circ}$ a través de la técnica de redes semánticas.

3. Diseñar un taller con la finalidad de mejorar el rendimiento académico en el tema de expresiones algebraicas y resolución de ecuaciones lineales en alumnos de $3^{\circ}$.

4. Implementar el taller para analizar el impacto en el rendimiento académico de alumnos de $3^{\circ}$ de secundaria.

\section{Propuesta}

La propuesta fue diseñar un taller, al que se llamó "Juguemos con Álgebra”, basado en la implementación de actividades lúdicas, con la finalidad de mejorar el rendimiento académico en los temas de expresiones algebraicas y resolución de ecuaciones lineales, así como la conceptualización de la materia de matemáticas en alumnos de $3^{\circ}$ de secundaria de la escuela Alberto Larios Villalpando T.V.

La muestra fue seleccionada tomando como referencia los resultados de la prueba ENLACE 2012, donde los alumnos de tercer grado fueron aquellos con quienes se llevó a cabo la intervención.

Dicho taller fue evaluado con la aplicación de un pre-test y un pos-test (con reactivos tomados de ENLACE 2012), además de la implementación de la técnica de redes semánticas.

\section{Hipótesis}

Con la implementación del taller "Juguemos con Álgebra" mejorará el rendimiento académico de los estudiantes de $3^{\circ}$ de secundaria en el tema de expresiones algebraicas y resolución de ecuaciones lineales, así como la conceptualización que se tiene de la materia de matemáticas. 


\section{Variables}

\section{Dependientes:}

Rendimiento académico en el tema de expresiones algebraicas y resolución de ecuaciones lineales, el cual fue evaluado con un pre-test y un post-test, ambos creados con reactivos de la prueba ENLACE 2012.

Conceptualización de la clase de matemáticas, que fue evaluada con la técnica de redes semánticas.

\section{Independiente:}

Taller "Juguemos con Álgebra", implementado con alumnos de 3er grado grupo A de la escuela secundaria Alberto Larios Villalpando T.V., de la ciudad de Colima, México, aplicado en siete sesiones, una vez por semana con una hora clase los días miércoles de 16:50 a 17:50 h., y la última semana trabajando dos sesiones, los días miércoles 5 y jueves 6 de diciembre. El objetivo del taller fue la mejora del rendimiento académico en el tema de álgebra y la modificación de la conceptualización de la materia de matemáticas.

\section{Participantes}

El grupo con el que se trabajó estuvo conformado por 16 alumnos de los cuales 9 eran mujeres y 7 hombres; el rango de edad se encontraba entre los 14 y 15 años. Los participantes fueron elegidos según sus resultados obtenidos en la prueba ENLACE 2012, siendo los de más bajo puntaje de su escuela.

\section{Diseño de investigación}

El enfoque es cuanti-cualitativo. El diseño es cuasi-experimental debido a que "se controlan y manipulan deliberadamente las condiciones que determinan los hechos en los que se trabaja, para después observar los efectos que se producen" (Escribano, 2004: 348), en este caso los hechos que se trabajaron pertenecen a la variable independiente que es el taller "Juguemos con Álgebra", evaluando sus efectos en el rendimiento académico y la conceptualización de la materia de matemáticas.

Por otra parte pertenece al diseño transeccional correlacional porque se describe la relación entre la variable independiente y la variable dependiente (Ortiz, 2003).

\section{Técnicas e instrumentos}

\section{Redes semánticas:}

Para evaluarlas se entregó una ficha a cada alumno, dándole indicaciones de que definieran con al menos cinco palabras sueltas y con la mayor precisión posible la palabra que aparecía en la ficha, en este caso matemáticas; una vez definida, se les solicitó que jerarquizaran las palabras definidoras dependiendo según su importancia.

Pre test:

El pre-test fue creado con 10 reactivos tomados de la prueba ENLACE 2012 del eje temático sentido numérico y pensamiento algebraico, debido a que en el taller sólo se trabajó con temas específicos de álgebra (Lenguaje algebraico y resolución de ecuaciones lineales). 


\section{Post test:}

Post-test fue creado con 10 reactivos similares a los del pre-test. Tanto con el pre test y el post test se evaluó el rendimiento académico.

\section{Resultados}

Con la finalidad de poder comprobar la hipótesis, en este apartado se analizaron dos aspectos principales para la investigación; el primero hace alusión a las diferencias arrojadas con la técnica de redes semánticas en un primer y segundo momento. El segundo aspecto analizado tiene que ver con las diferencias encontradas en el rendimiento académico, entre el pre-test y el pos-test.

\section{Diferencias arrojadas con la técnica de redes semánticas:}

\section{Conjunto SAM del concepto de Matemáticas}

Tabla 1. Resultados de la primera aplicación de redes semánticas

\begin{tabular}{|l|c|c|}
\hline \multicolumn{1}{|c|}{ Palabra definidora } & Valor M Total & Valor FMG (\%) \\
\hline Números & 107 & 100 \\
\hline Suma & 79 & 73.83 \\
\hline División & 67 & 62.62 \\
\hline Resta & 65 & 60.75 \\
\hline Multiplicación & 57 & 53.27 \\
\hline Problemas & 41 & 38.32 \\
\hline Pensar & 38 & 35.51 \\
\hline Operaciones & 37 & 34.58 \\
\hline Álgebra & 23 & 21.50 \\
\hline Signos & 22 & 20.56 \\
\hline Cuentas & 18 & 16.82 \\
\hline Ecuaciones & 18 & 16.82 \\
\hline Binomios & 18 & 16.82 \\
\hline Complicado & 14 & 13.08 \\
\hline Resultado & 13 & 12.15 \\
\hline \multicolumn{2}{|l|}{} \\
\hline
\end{tabular}

Fuente: Elaboración propia, 2013.

Tabla 2. Resultados de la segunda aplicación de redes semánticas

\begin{tabular}{|l|c|c|}
\hline Palabra definidora & Valor M Total & Valor FMG (\%) \\
\hline Diversión & 60 & 100 \\
\hline Juegos & 58 & 96.67 \\
\hline Ecuaciones & 39 & 65 \\
\hline Multiplicación & 34 & 56.67 \\
\hline
\end{tabular}


NÚÑEZ RODRÍGUEZ ET AL.: LA IMPLEMENTACIÓN DEL TALLER “JUGUEMOS CON ÁLGEBRA”...

\begin{tabular}{|l|c|c|}
\hline Palabra definidora & Valor M Total & Valor FMG (\%) \\
\hline Problemas & 25 & 41.67 \\
\hline Sumas & 25 & 41.67 \\
\hline Divisiones & 25 & 41.67 \\
\hline Expresiones algebraicas & 25 & 41.67 \\
\hline Aprendizaje & 24 & 40 \\
\hline Incógnita & 19 & 31.67 \\
\hline Chidas & 19 & 31.67 \\
\hline Restas & 17 & 28.33 \\
\hline Buena & 17 & 28.33 \\
\hline Binomios & 16 & 26.67 \\
\hline Álgebra & 16 & 26.67 \\
\hline \multicolumn{2}{|c|}{} \\
\hline
\end{tabular}

Fuente: Elaboración propia, 2013.

Para la interpretación de los datos fue necesario agrupar las palabras definidoras (ver tabla 1 y 2) en las siguientes cuatro categorías: descripción de la materia, descripción de la clase, emociones positivas y emociones negativas. Debido a que la técnica de redes semánticas pretende conocer el significado psicológico que las personas le otorgan a una palabra, y puesto que la investigación pretende analizar la actitud que los estudiantes tienen respecto a la palabra matemáticas, las categorías de emociones positivas y negativas serán aquellas que nos ayudarán a cumplir con nuestro objetivo de investigación quedando las categorías de descripción de la materia y descripción de la clase como categorías neutras.

Una vez categorizadas las palabras definidoras, podemos observar que existe un cambio notorio en las emociones positivas y negativas, puesto que en la primer aplicación no existía ninguna palabra que entrara dentro de las emociones positivas y teniendo una dentro de las emociones negativas y en la segunda aplicación se hizo notorio el incremento de las emociones positivas y la anulación de emociones negativas (ver tablas 3 y 4).

Tabla 3. Categorías de la primera aplicación

\begin{tabular}{|l|c|}
\hline Descripción de la materia & 13 \\
\hline Descripción de la clase & 1 \\
\hline Emociones negativas & 1 \\
\hline Emociones positivas & 0 \\
\hline
\end{tabular}

Fuente: Elaboración propia, 2013.

Tabla 4. Categorías de la segunda aplicación

\begin{tabular}{|l|c|}
\hline Descripción de la materia & 10 \\
\hline Descripción de la clase & 2 \\
\hline Emociones negativas & 0 \\
\hline Emociones positivas & 3 \\
\hline
\end{tabular}

Fuente: Elaboración propia, 2013. 
Analizando las palabras que entraron en cada una de las categorías neutras podemos notar que en la primera aplicación obtuvimos un mayor número de palabras definidoras que puntualizan la categoría de descripción de la materia, disminuyendo estas mismas en una segunda aplicación y notándose la incorporación de dos palabras relacionadas con los temas abordados en el taller Juguemos con Álgebra: “incógnita y expresiones algebraicas” (ver tabla 5).

Tabla 5. Primera categoría (descripción de la materia)

\begin{tabular}{|c|c|c|c|}
\hline \multicolumn{2}{|c|}{ Primera aplicación } & \multicolumn{2}{|c|}{ Segunda aplicación } \\
\hline Números & Álgebra & Suma & Incógnita \\
\hline Suma & Signos & Divisiones & Álgebra \\
\hline División & Cuentas & Restas & Binomio \\
\hline Resta & Ecuaciones & Multiplicación & Ecuaciones \\
\cline { 1 - 2 } Multiplicación & Binomios & Problemas & $\begin{array}{c}\text { Expresiones al- } \\
\text { gebraicas }\end{array}$ \\
\cline { 1 - 2 } Problemas & Resultado & &
\end{tabular}

Fuente: Elaboración propia, 2013.

Ahora bien, basándonos en la siguiente categoría: "descripción de la clase" podemos notar que a pesar de considerarse como una categoría neutra, nos arroja datos interesantes, debido a que las palabras obtenidas durante la primera y segunda aplicación dan un giro importante al considerarse en un primer instante como el solo hecho de pensar a la existencia de juegos y aprendizaje en un segundo momento, donde podemos apreciar que el juego es considerado como una herramienta para lograr el aprendizaje (ver tabla 6).

Tabla 6. Segunda categoría (descripción de la clase)

\begin{tabular}{|c|c|}
\hline Primera aplicación & Segunda aplicación \\
\hline \multirow{2}{*}{ Pensar } & Juegos \\
\cline { 2 - 2 } & Aprendizaje \\
\hline
\end{tabular}

Fuente: Elaboración propia, 2013.

\section{Diferencias encontradas entre los resultados del pre-test y pos-test:}

Una vez evaluada la conceptualización a través de las redes semánticas, pasamos a la parte del rendimiento académico, el cual se evaluó en un primer momento antes de la aplicación del taller "Juguemos con Álgebra" con un pre-test y en un segundo momento, después de la implementación del taller con un post-test, ambos creados con reactivos de la prueba ENLACE.

A continuación se muestran los resultados que nos ayudarán a comparar la mejora del rendimiento académico:

Tabla 7. Resultado de pre-test y post-test

\begin{tabular}{|c|c|c|}
\hline Nombre: & Pre-test & Post-test \\
\hline Estudiante 1 & 1 & 2 \\
\hline Estudiante 2 & 0 & 1 \\
\hline Estudiante 3 & 0 & 3 \\
\hline Estudiante 4 & 2 & 3 \\
\hline Estudiante 5 & 3 & 3 \\
\hline Estudiante 6 & 0 & 4 \\
\hline
\end{tabular}


NÚÑEZ RODRÍGUEZ ET AL.: LA IMPLEMENTACIÓN DEL TALLER “JUGUEMOS CON ÁLGEBRA”...

\begin{tabular}{|c|c|c|}
\hline Nombre: & Pre-test & Post-test \\
\hline Estudiante 7 & 3 & 2 \\
\hline Estudiante 8 & 4 & 5 \\
\hline Estudiante 9 & 6 & 1 \\
\hline Estudiante 10 & 3 & 4 \\
\hline Estudiante 11 & 0 & 4 \\
\hline Estudiante 12 & 3 & 3 \\
\hline Estudiante 13 & 1 & 3 \\
\hline Estudiante 14 & 1 & 3 \\
\hline Estudiante 15 & 2 & 3 \\
\hline Estudiante 16 & 4 & 7 \\
\hline PROMEDIO GRUPAL & 2.06 & 3.19 \\
\hline
\end{tabular}

Fuente: Elaboración propia, 2013.

Como podemos ver en la tabla 10, es evidente el incremento del promedio, aunque sigue siendo una calificación reprobatoria; el aumento se hace notar a simple vista, pero esto no nos asegura que sea favorable o significativo para nuestro objetivo de investigación, para ello se hizo necesario realizar la prueba de confiabilidad Wilcoxon.

Para llevar a cabo la prueba de Wilcoxon es necesario tener la diferencia entre el pre-test y el pos-test de cada alumno, esto es, a la calificación obtenida en el pos-test le resta la calificación del pre-test (ver tabla 8).

Tabla 8. Diferencia entre pre-test y post-test

\begin{tabular}{|c|c|c|}
\hline Pre-test & Post-test & Diferencia \\
\hline 1 & 2 & 1 \\
\hline 0 & 1 & 1 \\
\hline 0 & 3 & 3 \\
\hline 2 & 3 & 1 \\
\hline 3 & 3 & 0 \\
\hline 0 & 4 & 4 \\
\hline 3 & 2 & -1 \\
\hline 4 & 5 & 1 \\
\hline 6 & 1 & -5 \\
\hline 3 & 4 & 1 \\
\hline 0 & 4 & 4 \\
\hline 3 & 3 & 0 \\
\hline 1 & 3 & 2 \\
\hline 1 & 3 & 2 \\
\hline 2 & 3 & 1 \\
\hline 4 & 7 & 3 \\
\hline
\end{tabular}

Fuente: Elaboración propia, 2013.

Una vez obtenidas las diferencias, se asigna un rango de diferencia, donde primeramente se descartan los alumnos que tuvieron diferencias de cero y se toman al resto con su valor absoluto, en seguida, se acomodan en orden de menor a mayor y si se repiten los valores, se obtiene un promedio entre las posiciones. Por ejemplo, existen 7 alumnos los cuales su diferencia fue de 1 , 
entonces sus posiciones serían 1, 2, 3, 4, 5, 6 y 7, con ellas obtenemos un promedio, en este caso 4 , y este será el rango de diferencia de los alumnos que tienen como diferencia 1 (ver tabla 9).

Tabla 9. Diferencias y rango de diferencia

\begin{tabular}{|c|c|}
\hline Diferencia & Rango de diferencia \\
\hline 1 & 4 \\
\hline 1 & 4 \\
\hline 3 & 10.5 \\
\hline 1 & 4 \\
\hline 0 & \\
\hline 4 & 12.5 \\
\hline-1 & 4 \\
\hline 1 & 4 \\
\hline-5 & 14 \\
\hline 1 & 4 \\
\hline 4 & 12.5 \\
\hline 0 & \\
\hline 2 & 8.5 \\
\hline 2 & 8.5 \\
\hline 1 & 4 \\
\hline 3 & 10.5 \\
\hline
\end{tabular}

Fuente: Elaboración propia, 2013.

Después de haber sido asignados los rangos de diferencias, se suman los correspondientes a las diferencias positivas y los de las diferencias negativas (tabla 10):

Tabla 10. Suma de rangos de las diferencias

\begin{tabular}{|c|c|}
\hline Positivos & Negativos \\
\hline 87 & 18 \\
\hline
\end{tabular}

Fuente: Elaboración propia, 2013.

Ahora de estos dos valores, nos quedamos con el menor y utilizamos la tabla asignada para comprobar si nuestros resultados son significativos.

Dado que nuestra muestra (n) era de 16 y se descartaron 2 cuya diferencia fue cero, ésta se redujo a 14 , teniendo un nivel de significancia del 0.02 lo cual nos dice que los resultados obtenidos en el post-test tienen un alto nivel de significancia para el taller (ver tabla 11) 
Tabla 11. Valores críticos en la prueba de Wilcoxon

\begin{tabular}{|c|c|c|c|c|c|c|}
\hline & 0.10 & 0.05 & 0.02 & 0.01 & 0.005 & 0.001 \\
\hline 5 & $0-15$ & - & - & - & - & - \\
6 & $2-19$ & $0-21$ & - & - & - & - \\
7 & $3-25$ & $2-26$ & $0-28$ & - & - & - \\
\hline 8 & $5-31$ & $3-33$ & $1-35$ & $0-36$ & - & - \\
9 & $8-37$ & $5-40$ & $3-42$ & $1-44$ & $0-45$ & - \\
10 & $10-45$ & $8-47$ & $5-50$ & $3-52$ & $1-54$ & - \\
\hline 11 & $13-53$ & $10-56$ & $7-59$ & $5-61$ & $3-63$ & $0-66$ \\
12 & $17-61$ & $13-65$ & $9-69$ & $7-71$ & $5-73$ & $1-77$ \\
13 & $21-70$ & $17-74$ & $12-79$ & $9-82$ & $7-84$ & $2-89$ \\
\hline 14 & $25-80$ & $21-84$ & $15-90$ & $12-93$ & $9-96$ & $4-101$ \\
\hline 15 & $30-90$ & $25-95$ & $19-101$ & $15-105$ & $12-108$ & $6-116$ \\
\hline
\end{tabular}

Fuente: Elaboración propia, 2013.

\section{Conclusiones}

Considerando como principal problema el rechazo que los alumnos de secundaria presentan por la materia de matemáticas, se buscó recurrir a una estrategia que ayudara a modificar su perspectiva sobre ésta, para tratar de mejorar su rendimiento académico en temas específicos de álgebra, donde éstos presentan mayor dificultad al no comprender el porqué de su expresión y el para qué de su aplicación, para ello se planteó la siguiente hipótesis:

Con la implementación del taller "Juguemos con Álgebra" mejorará el rendimiento académico de los estudiantes de $3^{\circ}$ de secundaria en el tema de expresiones algebraicas y resolución de ecuaciones lineales, así como la conceptualización que se tiene de la materia de matemáticas.

Por lo tanto, después de haber analizado los resultados obtenidos y después de la aplicación del taller, podemos aceptar la hipótesis establecida en la investigación.

Lo anterior, puesto que se logró modificar la perspectiva que los alumnos tenían acerca de la materia de matemáticas, cambiando la idea de ser una clase en la que solo requerían pensar, a una clase en la que mientras jugaban, aprendían, lográndose la mejora del rendimiento académico al obtenerse un alto nivel de significancia (en el pre y post test), al analizarse con la prueba Wilconxon, cumpliéndose así el objetivo general de la investigación.

Por otro lado, hay que destacar que durante las sesiones del taller siempre se buscó seguir lo propuesto por el Programa de Estudios 2011 donde hace mención a un enfoque didáctico apoyado en el trabajo colaborativo que implica la interacción entre alumnos y maestros, llegándose a lo que menciona Barnett (2003): las personas no aprendemos solas, si no que estamos integradas en un contexto social que da sentido a lo que aprendemos.

De la misma forma, en las actividades con las que se trabajó se consideró al juego como una estrategia, cuyo principal objetivo fue modificar la conceptualización que los alumnos tienen de la materia, como lo establece García (1998) desde el punto vista psicopedagógico, el juego se valora como un elemento que favorece a todo tipo de aprendizajes, puede proporcionar al niño una gran variedad de experiencias y estímulos, todo un bagaje de vivencias útiles y necesarias para su adecuado desarrollo.

El trabajo presentado, muestra como al modificar, la manera de recibir las clases cambia su conceptualización de la materia y favorece el rendimiento de la misma. El trabajo nos deja un rica y valiosa lección sobre, como deben de ser nuestra acción docentes, para lograr que nuestros alumnos tengan una mejor y enriquecedora visión de lo que son los matemáticas, logrando que al no desagradarles la materia, favorezca su rendimiento académico. 


\section{Sugerencias}

De acuerdo a lo analizado en la presente investigación se sugiere:

- Llevar un seguimiento y control con las bitácoras de observación de los alumnos, del observador externo y de los facilitadores para realizar un mejor diseño de cada una de las sesiones.

- Aumentar el número de sesiones por semana para poder profundizar en los temas que presenten mayor rezago en los alumnos.

- Aumentar el tiempo en cada sesión, mínimo dos horas clase.

- Buscar el apoyo de la institución y de los padres de familia para que los alumnos asistan a todas las sesiones.

- Apoyo por parte del maestro titular de la materia para que retome en su clase, los temas abordados en el taller. 


\section{REFERENCIAS}

Alarcón, J., Bonilla, E., Nava, R., Rojano, T., y Quintero, R. (1994). Libro del maestro (2001. ${ }^{\text {a }}$ ed.). México, DF: Secretaria de Educación pública. Recuperado de http://www.reforma secundaria.sep.gob.mx/matematicas/pdf/orientaciones/libromaestro.pdf

Barnett, L. (2003). Motivación, tratamiento de la diversidad y rendimiento académico : el aprendizaje cooperativo. Venezuela: Editorial Laboratorio Educativo.

Chacón, M. (2005). Educación física para niños con necesidades educativas especiales (primera.). San José, Costa Rica: Universidad Estatal a Distancia.

Escribano, A. (2004). Aprender a enseñar : fundamentos de didáctica general. Cuenca: Ediciones de la Universidad de Castilla-La Mancha.

García, A. (2002). Los Juegos en la educación física de los 12 a los 14 años : primer ciclo de la ESO. Barcelona: INDE.

Jiménez, J., Rodríguez, M., y Estrada, R. (2006). Matemáticas 1 SEP (2006. ${ }^{a}$ ed.). Umbral.

Martínez, V. (2007). La buena educación : reflexiones y propuestas de psicopedagogía humanista. Rubí, Barcelona: Anthropos Editorial.

Ortiz, F. (2003). Diccionario de metodología de la investigación científica. México: Limusa, Noriega.

Sánchez, A., Gómez, C. y de la Cruz, E. (2010, septiembre). El impacto de un taller basado en la teoría de situaciones didácticas para mejorar la habilidad de emplear el lenguaje algebraico en alumnos de $2^{\circ}$ grado de secundaria. Universidad de Colima, Colima, Colima, México.

SEP (2011). Programas de Estudio 2011. Guía para el maestro. Matemáticas. México: Secretaria de Educación Pública. Recuperado de http://basica.sep.gob.mx/dgdc/sitio/pdf/ inicio/matlinea/2011/Matematicas_SEC.pdf

- (2012). Evaluación Nacional del Logro Académico en Centros Escolares. México: Secretaria de Educación Pública. Recuperado de http://www.enlace.sep.gob.mx/ba/

Stigol, C. (2012). Conceptualización: saber explicar, orientar, encauzar y seleccionar. Recuperado de www.revistameyerhold.com/revista1/ CONCEPTUALIZACION.doc

Trueba, B. (1999). Talleres integrales en educación infantil : una propuesta de organización del escenario escolar. Madrid: Ediciones de la Torre.

Valdez, J. (2004). Las redes semánticas naturales, uso y aplicaciones en psicología social. México.

\section{SOBRE LOS AUTORES}

Ana Laura Núñez Rodríguez: Estudiante de la Facultad de Ciencias de la Educación, perteneciente a la Universidad de Colima, que actualmente se encuentra cursando el octavo semestre de la licenciatura en educación media especializada en matemáticas. Ha participado como tutor en el programa organizado por la universidad de Colima, Peraj - Adopta un amig@, el cual consistió en servir como apoyo y guía para un alumno de educación primaria en el ámbito personal y educativo. Además, ha realizado sus prácticas profesionales en diversas escuelas secundarias del estado de Colima, así como en el bachillerato del municipio de Coquimatlán, y ha colaborado con estas escuelas en la implementación de asesorías para sus estudiantes.

Alma Minerva Aguilar Martínez: Estudiante de la Facultad de Ciencias de la Educación, perteneciente a la Universidad de Colima, que actualmente se encuentra cursando el octavo semestre de la Licenciatura en Educación Media especializada en Matemáticas. Ha participado como 
tutor en el programa organizado por la universidad de Colima, Peraj - Adopta un amig@, el cual consistió en servir como apoyo y guía para un alumno de educación primaria en el ámbito personal y educativo.

Mireya Sarahí Abarca Cedeño: Licenciada en Psicología y en Educación Especial en el Área de Problemas de Aprendizaje; Maestra en Ciencias en el Área de Psicología Aplicada, por la Universidad de Colima. Actualmente cursando el Doctorado en Educación Física y Artística en la Universidad de Extremadura, España. Profesora e Investigadora de Tiempo Completo en la Facultad de Ciencias de la Educación de la Universidad de Colima; su línea de investigación es Modelos Educativos. Ha impartido múltiples cursos, talleres y realizado publicaciones el área de educación ambiental, modelos educativos, educación a través del arte, entre otros

Jesús Antonio Larios Trejo: Licenciado en Educación Media, especializado en Matemáticas. Actualmente se encuentra cursando la Maestría en Educación por parte de la Universidad Autónoma de Guadalajara. Cuenta con diversas investigaciones y proyectos. Labora en la Universidad de Colima como profesor de la Licenciatura en Educación Media con la especialidad en Matemáticas; y como docente frente a grupo de la Secundaria Técnica "Moisés Sáenz Garza". Ha impartido múltiples cursos sobre la enseñanza de las matemáticas y la educación en matemáticas. 